Full Length Article

\title{
Passivation and dissolution mechanisms in ordered anodic tantalum oxide nanostructures
}

\author{
C.F. Almeida Alves ${ }^{\mathrm{a}, \mathrm{b}, *}$, S. Calderon V. ${ }^{\text {, P.J. Ferreira }}{ }^{\mathrm{b}, \mathrm{c}, \mathrm{d}}$, L. Marques ${ }^{\mathrm{e}}$, S. Carvalho ${ }^{\mathrm{a}, \mathrm{f}}$

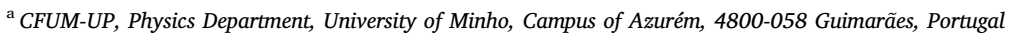 \\ ${ }^{\mathrm{b}}$ INL - International Iberian Nanotechnology Laboratory, Av. Mestre José Veiga s/n, 4715-330 Braga, Portugal \\ ${ }^{\mathrm{c}}$ Mechanical Engineering Department and IDMEC, Instituto Superior Técnico, University of Lisbon, Av. Rovisco Pais, 1049-001 Lisboa, Portugal \\ ${ }^{\mathrm{d}}$ Materials Science and Engineering Program, University of Texas at Austin, Austin, TX 78712, USA \\ e CFUM-UP, Physics Department, University of Minho, Campus of Gualtar, 4710-057 Braga, Portugal \\ ${ }^{\mathrm{f}}$ SEG-CEMMPRE, Mechanical Engineering Department, University of Coimbra, 3030-788 Coimbra, Portugal
}

\section{A R T I C L E I N F O}

\section{Keywords:}

Tantalum

Anodization

Nano-dimples

STEM

EDS

\begin{abstract}
A B S T R A C T
Tantalum oxide $\left(\mathrm{Ta}_{2} \mathrm{O}_{5}\right)$ nanostructures exhibit outstanding electrical and optical properties, as well as, high chemical resistance and stability. These materials have great potential for biomedical, catalysis, semiconductors and energy applications due to their large surface area and high specific charge, when arranged in nanoporous or nanotubular morphologies. In order to obtain these structures, an anodization process, which is inexpensive, reproducible and easy to scale up, is used. Yet, depending on the anodization conditions, the formation of a nanoporous or nanotubular layer is difficult to stabilize during the anodization process. In this regard, anodized tantalum oxide nanostructures were produced to understand the effect of the anodization conditions, including electrolyte concentration, potential and time. The nanopores or nanotubes morphologies, their chemical composition and structure were investigated by FIB-SEM, double-corrected TEM-STEM and EDS. We found that it is necessary to have high acid concentrations (mixture of $\mathrm{H}_{2} \mathrm{SO}_{4}$ with $\mathrm{HF}$ ) to be able to form nanoporous or nanotubular structures. Despite the capacity of $\mathrm{HF}$ to dissolve and create anodic oxide nanostructures, the amount of $\mathrm{H}_{2} \mathrm{SO}_{4}$ concentration in the mixture is very important, leading to a dimple morphology. Furthermore, the increase of the anodization potential/electrical field clearly leads to an increase in the dimples diameter.
\end{abstract}

\section{Introduction}

Nanoscale patterned surfaces have been addressed in a variety of fields [1] due to their interest for catalysis, electronics, sensors, energy storage and biomedical sciences [2]. In particular, a significant effort has been focused on controlling and manipulating the arrays order [3], since large surface areas and three dimensional (3D) guides for the growth of nanomaterials are some of the advantages associated with well-ordered nanostructured surfaces [4]. This is the case of using electrochemical anodization for the growth of nanostructures, which has been studied for more than 50 years. In this regard, it is well reported in the literature that the formation of nanostructures obtained by anodization are affected by the electrolyte composition and concentration, the applied potential and the anodization time $[3,5]$. The application of an electric field favors the ionic transport of $\mathrm{O}^{2-}$ and $\mathrm{OH}^{-}$. As a result, the thickness of the anodic layer increases as a function of the anodization time $[3,4,6]$. Furthermore, a voltage increase leads to higher anodic current densities, thus accelerating the anodization rate.
The electrolyte is frequently composed by strong acids, such as $\mathrm{H}_{2} \mathrm{SO}_{4}$, $\mathrm{HF}$ or $\mathrm{H}_{3} \mathrm{PO}_{4}$, where the efficiency and anodization rate is determined by their concentrations. Thus, the electrolyte selection affects the formation of pores, which is specific for each material [7].

Among various nanostructured surfaces, tantalum oxide have great technological interest due to its excellent electrical and optical properties, such as high transparency, high refractive index $(\mathrm{n} \approx 2.2$ at $633 \mathrm{~nm}$ ) and high dielectric constant $(\varepsilon \mathrm{r} \approx 25)$ [8-11], which is very promising for electronic and sensor applications.

The anodization of Ta leads to the formation of a dense anodic oxide layer, usually $\mathrm{Ta}_{2} \mathrm{O}_{5}$ [3]. This anodic layer reveals specific characteristics, such as high corrosion resistance and inert behavior, responsible for high resistance to chemical attack $[12,13]$. On the other hand, some authors reported that the formation of an ordered nanoporous layer is possible with a mixture of sulphuric and hydrofluoric acids $\left(\mathrm{H}_{2} \mathrm{SO}_{4}\right.$ and HF) $[2,4,7,14-17]$. Hany El-Sayed et al. [15] reported the formation of a highly ordered nanoporous layer with low potentials around $10-20 \mathrm{~V}$, which is below the $\mathrm{TaO}_{\mathrm{x}}$ breakdown potential $(\approx 200 \mathrm{~V})$, at anodization

\footnotetext{
* Corresponding author at: INL - International Iberian Nanotechnology Laboratory, Av. Mestre José Veiga s/n, 4715-330 Braga, Portugal.

E-mail address: cristiana.alves@inl.int (C.F. Almeida Alves).
} 
times varying between $30 \mathrm{~s}$ and $10 \mathrm{~min}$, using $\mathrm{H}_{2} \mathrm{SO}_{4}$ mixed with $\mathrm{HF}$ at high electrolyte concentrations (16.4 and $2.9 \mathrm{M}$, respectively).

The formation of a porous anodic layer, through anodization, results from a continuous process that involves a competition between the oxidation of the metal in the oxide/metal interface and dissolution of the oxide at the oxide/electrolyte interface $[3,4]$. However, to the authors' knowledge there is very little work that have demonstrated the oxidation and dissolution processes for the anodization of Ta. Several studies have suggested that pits are generated during the first seconds of anodization, after the formation of the compact anodic layer. The growth of pits due to the preferential radial spread promoted by fieldenhanced dissolution on small surface asperities led to the formation of ordered nanopores $[4,7,14,18]$. These papers suggested that the formation of the nanostructures (e.g. nanopores and/or nanotubes) by anodization was due to the passivation of the surface and subsequent dissolution.

In the current work, we provide evidence about the formation mechanism of $\mathrm{Ta}_{2} \mathrm{O}_{5}$ nanostructures by coupling aberration-corrected scanning transmission electron microscopy (STEM) and energy dispersive spectroscopy (EDS) mapping. A systematic methodology was used to determine the effect of passivation and dissolution in order to understand the growth of the nanostructures. Moreover, it is demonstrated the dependency of the anodization conditions, such as electrolyte concentration, applied voltage and anodization time, on the formation of the nanostructures.

\section{Materials and methods}

\subsection{Materials}

High-purity Ta sheets (99.95\% with $0.5 \mathrm{~mm}$ ) were purchased from Testbourne Ltd. $\mathrm{H}_{2} \mathrm{SO}_{4}(95-98 \% \mathrm{vol})$ and HF (48\%vol) ACS grade reagents were purchased from Sigma-Aldrich. All chemicals were used asreceived without any further purification, and all electrolytes were prepared with distillated water.

\subsection{Electrochemical anodization}

Ta specimens were degreased and cleaned in an ultrasonic bath during $5 \mathrm{~min}$ in benzene, followed by $5 \mathrm{~min}$ in ethanol. Subsequently, the samples were rinsed with distillated water and dried in air. Prior to the electrochemical anodization, an acid etching with a 1:1 volumetric mixture of $\mathrm{HNO}_{3}$ with $\mathrm{HF}$ acids was performed to make the surface smoother and enhance the anodic morphology homogeneity.

The electrochemical anodization was carried out in potentiostatic mode coupled with an Agilent N5751A DC Power supply and Agilent 34450A 5 1/2 Digit Multimeter. The anodization was performed in a two-electrode cell configuration, comprising a working electrode (Ta sheet) and a reference electrode (carbon stick). The distance between the electrodes was kept constant at $30 \mathrm{~mm}$, and the electrolyte was magnetically stirred during the anodization to promote its homogeneity in terms of composition and temperature. Ta specimens $\left(20 \times 20 \times 0.5 \mathrm{~mm}^{3}\right)$ were anodized in concentrated mixtures of 7-9 volumetric percentage (vol. \%) of $\mathrm{H}_{2} \mathrm{SO}_{4}, 0.125-1$ vol\% of $\mathrm{HF}$ and 0.5-2.5 vol\% of $\mathrm{H}_{2} \mathrm{O}$ under voltages between 15 and $50 \mathrm{~V}$ and times ranging between 5 and $120 \mathrm{~s}$ at room temperature. Immediately after the anodic treatment, the samples were rinsed with multiple immersions in distillated water and dried in air.

\subsection{Electron microscopy}

A FEI Nova 200 scanning electron microscope (SEM) operating at $5 \mathrm{keV}$ was used to observe the surface morphology of the anodic layer. Cross-section samples were prepared using a Helios 450S (FEI) focus ion beam for subsequent STEM/EDS analysis. Furthermore, a drop casting method was used to prepare TEM lacey carbon grids with distilled water that was used for cleaning the anodic surface, in order to analyze the anodic "residues" present in the cleaning solution. High angular annular dark field (HAADF) STEM images were acquired in a double-corrected FEI Titan microscope operated at $300 \mathrm{keV}$. The images were recorded using a convergence angle of $21 \mathrm{mrad}$ with a pixel dwell time set at $10 \mu \mathrm{s}$. A camera length of $115 \mathrm{~mm}$ was selected, which collects electrons between 56 and 200 mrad. Energy dispersive X-ray spectroscopy mapping (EDS-mapping) was performed in the same microscope, equipped with a Super-X EDS detector. Iterative maps were recorded with a dwell time per pixel of $10-20 \mu$ at $300 \mathrm{keV}$, a current of approximately $300-350 \mathrm{pA}$ and collection times of $10 \mathrm{~min}$.

\subsection{Image segmentation}

Fiji ImageJ software was used to determine the average diameter and distribution of the dimples over the Ta surface as a function of the applied potential. A set of image processing conditions, including circularity ( $>0.7$ ), were selected to study the samples. Then, a descriptive statistical analysis was performed. It is important to emphasize that when was not possible to distinguish between two or more different dimples due to low image contrast/resolution, these were not considered on the descriptive statistical analysis, as shown in Fig. 1.

Furthermore, the inter-dimple distance was estimated by using the ImageJ plugin "Nearest Neighbor Distances Calculation". In order to study the electric field influence on long-range order, the radial distribution function (RDF) was estimated considering the center of the dimples by using a Fiji ImageJ macro.

\section{Results and discussion}

Electrochemical anodization assisted by an electric field mechanism shows the formation of highly ordered nano-dimples arrays on Ta surfaces. Fig. 2 shows Ta anodized surfaces using different HF concentration (from 0.5 to 0.125 volumetric percentage (vol.\%)) with constant $\mathrm{H}_{2} \mathrm{SO}_{4}$ concentration (8 vol\%), at room temperature under $15 \mathrm{~V}$ during $120 \mathrm{~s}$.

The growth of nanostructures through anodization results from a competition between the continuous formation and dissolution of the anodic $\mathrm{TaO}_{\mathrm{x}}$. Thus, the ratio between the passivation and dissolution elements present in the electrolyte plays a definitive role in the formation of such nanostructures. In this regard, despite the formation of highly ordered nanostructures, in agreement with the literature [15], a dependence of the formation of the nanostructures on HF concentration is observed. Decreasing the HF concentration in the anodic electrolyte from 0.5 to 0.25 volumetric percentage (vol.\%) (Fig. 2a and b), while keeping constant the $\mathrm{H}_{2} \mathrm{SO}_{4}$ concentration ( $8 \mathrm{vol} \%$ ), leads to a situation

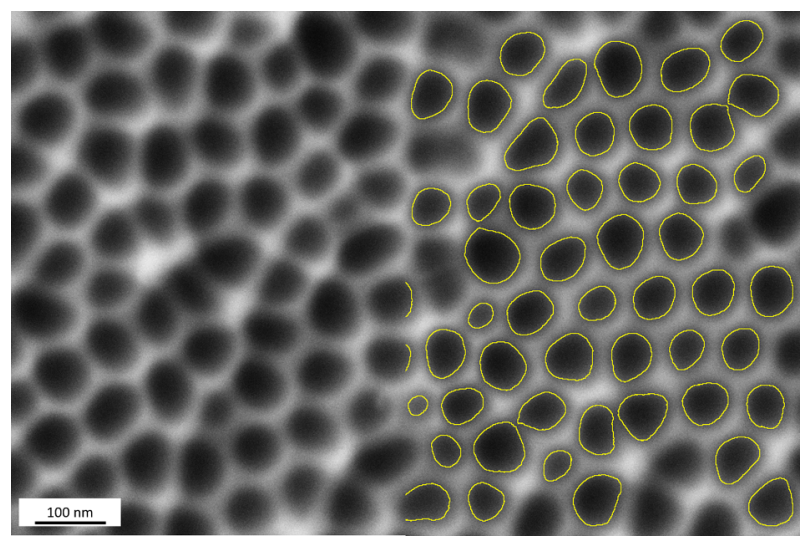

Fig. 1. Planar view secondary electron SEM image and corresponding image segmentation performed on a Ta sample anodized with 8:0.25 (vol:vol) of $\mathrm{H}_{2} \mathrm{SO}_{4}: \mathrm{HF}$, at room temperature under $30 \mathrm{~V}$ during $120 \mathrm{~s}$. 
a) $8 \mathrm{H}_{2} \mathrm{SO}_{4}: 0.5 \mathrm{HF}$

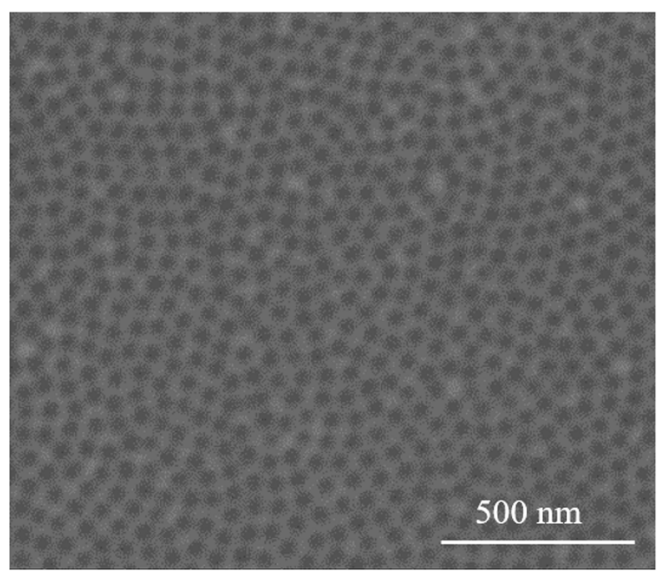

c) $8 \mathrm{H}_{2} \mathrm{SO}_{4}: 0.125 \mathrm{HF}$

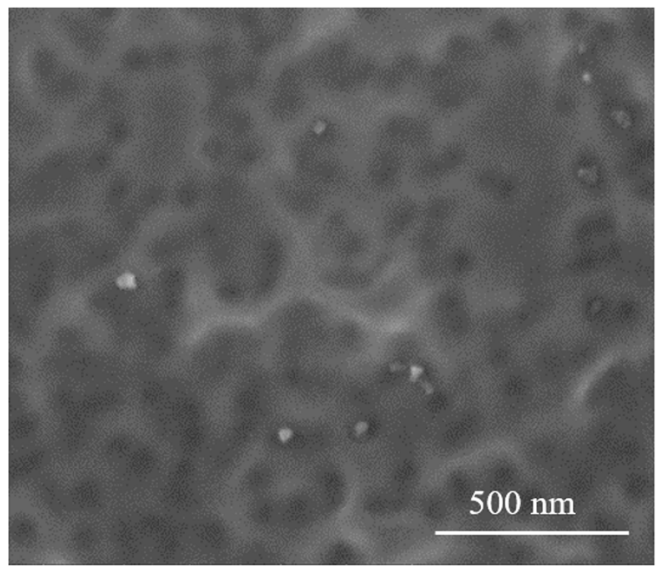

b) $8 \mathrm{H}_{2} \mathrm{SO}_{4}: 0.25 \mathrm{HF}$

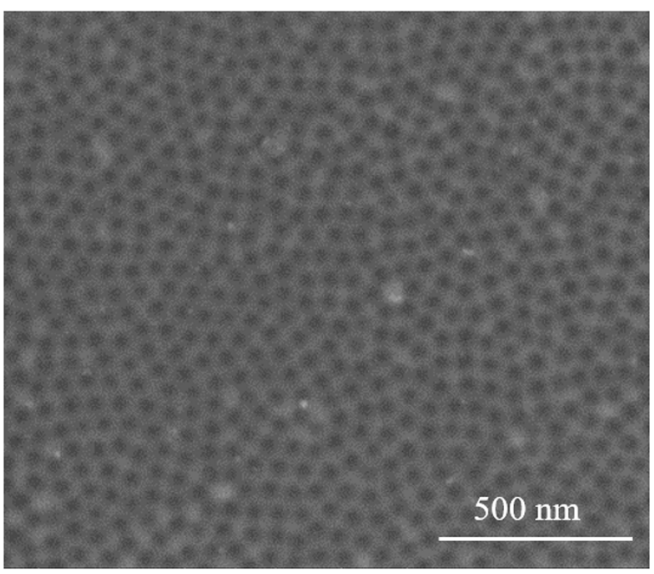

d)

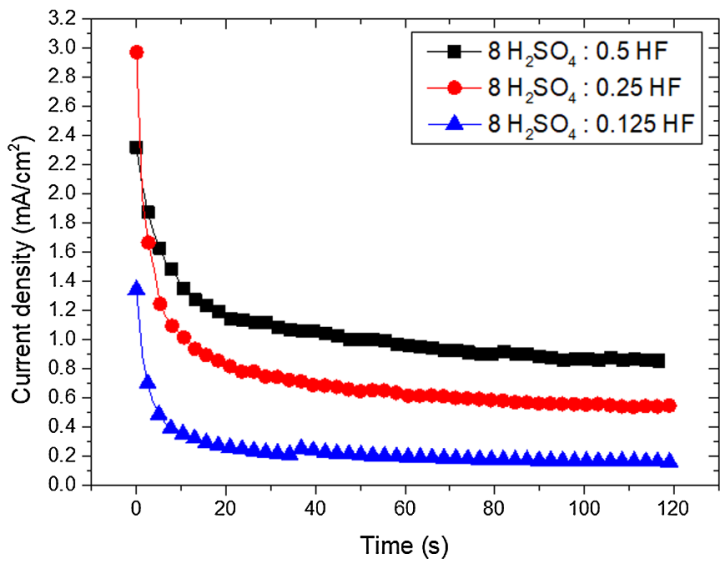

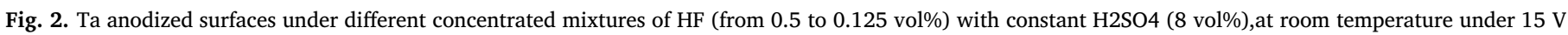
during $120 \mathrm{~s}$ : (a, b and c) Planar view secondary electron SEM images; (d) Current density vs. time anodization curve.

where the formation of nanostructures remains ordered. However, with a reduction of the $\mathrm{HF}$ concentration down to $0.125 \mathrm{HF}$ vol.\% (Fig. 2c) a dissolution limit of the anodic $\mathrm{TaO}_{\mathrm{x}}$ is reached having a dramatic effect on the growth of the nanostructures, particularly the arrays order. In fact, a clear reduction of the current density is observed for lower HF concentrations, showing the lower dissolution rate. In this process, $\mathrm{F}^{-}$ ions will attack the anodic oxide and dissolve it by pitting corrosion. A decrease in $\mathrm{F}^{-}$concentration, simultaneously with an increase in water content, stabilizes the anodic oxide, decreasing the dissolution [19].

Nevertheless, the formation of the nanostructures not only depends on the HF capacity to dissolve and create anodic oxide nanostructures, but also on the $\mathrm{H}_{2} \mathrm{SO}_{4}$ concentration to achieve highly ordered nanostructures. Fig. 3 shows the Ta anodized surfaces using different $\mathrm{H}_{2} \mathrm{SO}_{4}$ concentrations (from 9 to 7 vol\%), with a constant HF concentration ( $0.5 \mathrm{vol} \%)$, at room temperature under $15 \mathrm{~V}$ during $120 \mathrm{~s}$.

Decreasing the $\mathrm{H}_{2} \mathrm{SO}_{4}$ concentration in the anodic electrolyte from 9 to 7 vol\% (Fig. 3a-c), while keeping the constant HF concentration (0.5 $\mathrm{HF}$ vol.\%), for $7 \mathrm{H}_{2} \mathrm{SO}_{4}$ vol.\%, a different dissolution rate is observed. As shown in Fig. 3d, a significant decrease in the current density is observed by increasing the concentration of $\mathrm{H}_{2} \mathrm{SO}_{4}$. This fact, can be explained by the reduction of $\mathrm{HF}$ dissociation with increasing $\mathrm{H}_{2} \mathrm{SO}_{4}$ concentration and formation of fluorosulfuric acid (Eq. (1)), which leads to a reduction of reactive fluoride ions $\left(\mathrm{F}^{-}, \mathrm{HF}^{2-}\right)$ and a consequent decrease of the tantalum oxide dissolution rate [20,21]. Moreover, higher sulfuric acid concentrations also increase the solution viscosity and hinders the diffusion of fluoride-containing species (HF,
$\mathrm{F}^{-}$and $\mathrm{HF}^{2-}$ ) to the surface, further limiting the oxide dissolution rate [21].

$\mathrm{HF}+\mathrm{H}_{2} \mathrm{SO}_{4} \leftrightarrow \mathrm{HSO}_{3} \mathrm{~F}+\mathrm{H}_{2} \mathrm{O}$

On the other hand, the literature $[2,22]$ suggests that concentrated sulfuric acid electrolytes induce the incorporation of anions $\left(\mathrm{SO}_{4}{ }^{2-}\right)$ into the anodic oxide, causing a surface passivation, being responsible for enhancing the HF capacity to dissolve the anodic layer and allow the formation of the nanostructures.

To study in detail the morphology and composition of the nanostructures, STEM-EDS mapping was performed. Fig. 4 shows a representative HAADF STEM - EDS map of the sample anodized in 8:0.5 (vol:vol) of $\mathrm{H}_{2} \mathrm{SO}_{4}$ mixed with $\mathrm{HF}$, at room temperature under $15 \mathrm{~V}$ during $120 \mathrm{~s}$.

These cross-section images reveal an array of dispersed dimples (shown by the surface fluctuations represented by the green dotted line), exhibiting an oxide thickness around $5 \mathrm{~nm}$ (Fig. 4a and inset), whereas the EDS mapping only shows the presence of Ta and O, without any vestiges of $S$ and/or $F$ detected in the anodic layer.

In addition, although there is a clear dependence of both HF and $\mathrm{H}_{2} \mathrm{SO}_{4}$ concentrations to achieve ordered nanostructures, all the anodization conditions tested led to a dimple-shaped type morphology.

The literature reports that the pits are generated during the first seconds of anodization, leading to the formation of ordered nanopores $[4,7,14,18]$. It is also stated $[4,7,18,23,24]$ that during anodization Fions continuously react with the oxide layer being incorporated in the 
a) $9 \mathrm{H}_{2} \mathrm{SO}_{4}: 0.5 \mathrm{HF}$

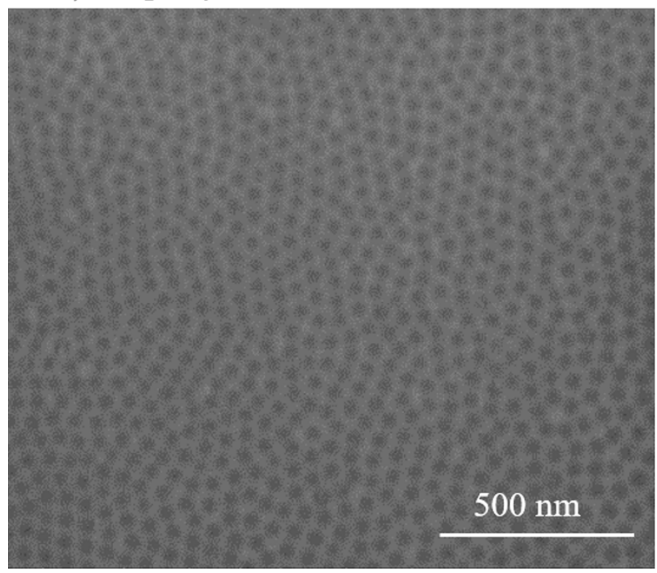

c) $7 \mathrm{H}_{2} \mathrm{SO}_{4}: 0.5 \mathrm{HF}$

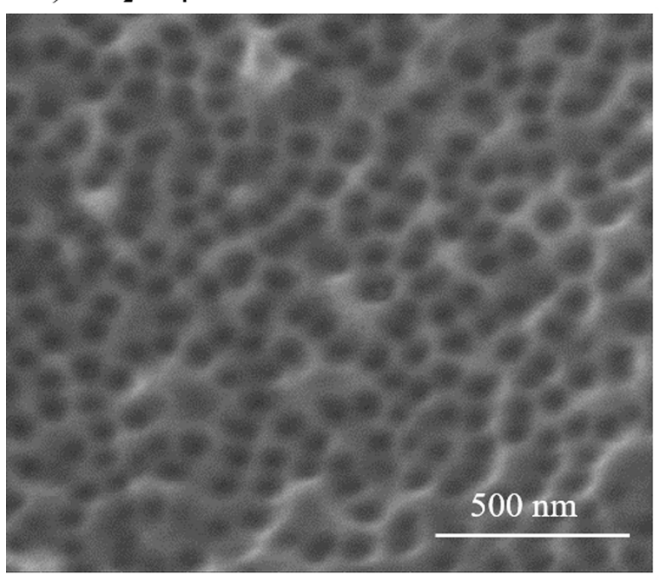

b) $8 \mathrm{H}_{2} \mathrm{SO}_{4}: 0.5 \mathrm{HF}$

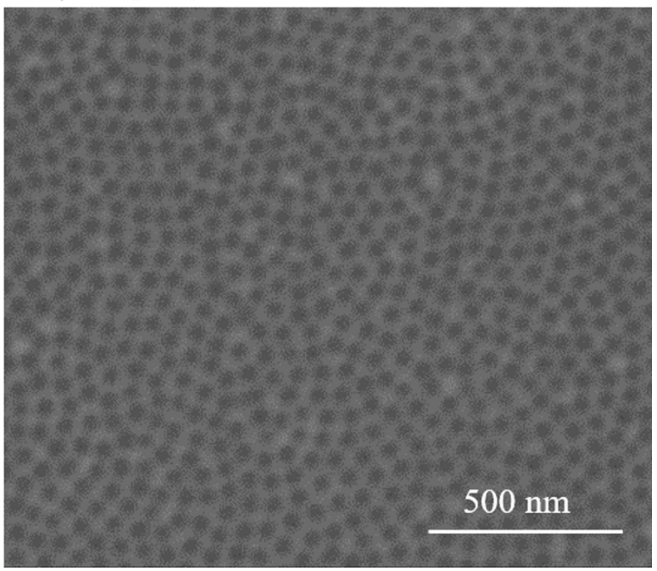

d)

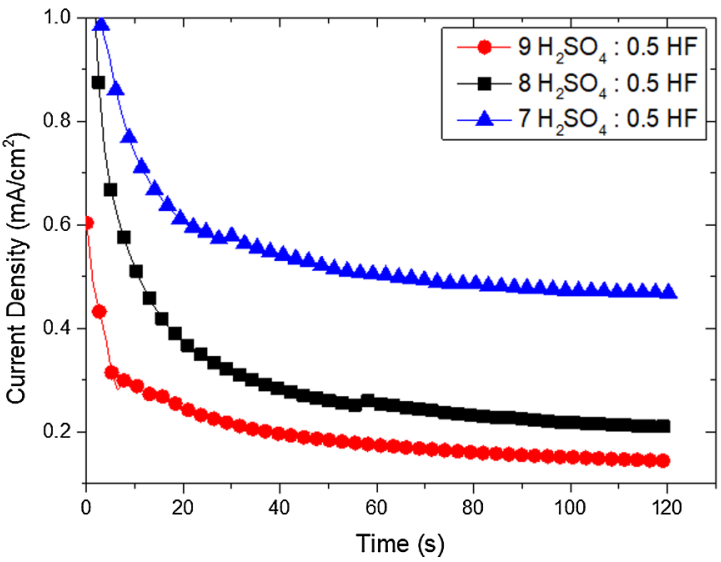

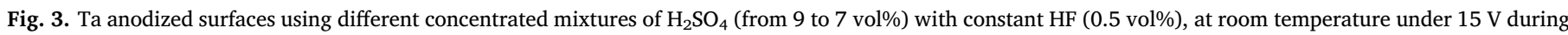
120 s: (a, b and c) Planar view secondary electron SEM images; (d) Current density vs. time anodization curve.

outer part of the anodic layer, forming $\mathrm{TaF}_{7}{ }^{2-}$ layer, according to the reaction presented in equation $2[19,25]$.

$2 \mathrm{Ta}+5 \mathrm{H}_{2} \mathrm{O} \rightarrow \mathrm{Ta}_{2} \mathrm{O}_{5}+10 \mathrm{H}^{+}+10 e^{-}$
$\mathrm{Ta}_{2} \mathrm{O}_{5}+14 \mathrm{~F}^{-}+10 \mathrm{H}^{+} \rightarrow 2 \mathrm{TaF}_{7}^{2-}+5 \mathrm{H}_{2} \mathrm{O}$

These metal-fluoride complexes are responsible for the growth and shape of the nanostructures [23]. Additionally, due to the higher migration velocity of the $\mathrm{F}^{-}$ions, compared with $\mathrm{O}^{2-}$ ions, the $\mathrm{F}^{-}$ions migrate from the outer part of the oxide layer to the inner part, being responsible for the separation of the pores, forming nanotubes due to its water solubility $[18,23]$.

In order to demonstrate the previous hypothesis, HAADF-STEM images (Fig. 5) and STEM-EDS compositional maps (Fig. 6) were acquired from the solid residues of the electrolyte after anodization.

First, it is important to notice that the solid residues of the anodization electrolyte shown in Fig. 5 demonstrate the existence of nanotubes in solution, revealing the formation of a nanotubular layer.

Fig. 6 shows the HAADF STEM and EDS spectrum images of a nanotube wall. Such nanotube is composed of Ta, $\mathrm{O}, \mathrm{S}$ and $\mathrm{F}$ indicating the presence of $\mathrm{SO}_{4}{ }^{2-}$ and/or $\mathrm{F}^{-}$ions on the nanotube walls. Fig. 6 also reveals that $\mathrm{S}$-rich zones exhibit less $\mathrm{F}$ content (see the $\mathrm{F} / \mathrm{S}$ ratio associated with the black and white area in Fig. 6.g) confirming that there is an ion exchange between $\mathrm{SO}_{4}{ }^{2-}$ and $\mathrm{F}^{-}$, which explains the effect of sulfuric acid concentration to achieve ordered nanostructures.

Furthermore, the surface anodized with the lowest $\mathrm{H}_{2} \mathrm{SO}_{4}$ concentration (Fig. 3.c) show a less ordered surface compared to the surfaces obtained with higher concentrations. However, the different topography is likely due to differences in the dissolution rates for each concentration. In fact, this effect is noticeable when comparing the residual nanotubes in solution after anodization for different concentrations (Fig. 5). As the dissolution rate for the sample anodized with 7:0.5 (vol:vol) of $\mathrm{H}_{2} \mathrm{SO}_{4}$ : $\mathrm{HF}$ is high, the surface dissolution becomes more unstable revealing a rougher surface.

Interestingly, the diameter and distribution of the dimples is independent of the anodization time, as shown in Fig. 7, where the morphology evolution of Ta anodized surfaces is maintained from $5 \mathrm{~s}$ to $60 \mathrm{~s}$.

Hany El-Sayed et al. [4], reported that the use of relatively high $\mathrm{H}_{2} \mathrm{SO}_{4}$ concentration leads to a more unstable process due to an enhanced incorporation of $\mathrm{SO}_{4}{ }^{2-}$ by the extremely acid electrolyte. Then, this effect may contribute for a dimple-type topography as shown in Fig. 7 inset for $60 \mathrm{~s}$, independently of the anodization runtime, thus explaining the common dimple-shaped morphology observed. In spite of the dimple-type topography, the nanotubes exhibit a low aspect ratio (Fig. 8a), which indicates that the nanotubes are mechanically instable. In Fig. 8 a can be seen an agglomerate of nanotubes that were released from the surface. It is worth noticing that grain boundaries in the Ta sheet (Fig. 8d) do not seem to increase the instability of the nanotubes, since the length of the nanotubes (Fig. 8a) in the solid residue does not correlate with the Ta sheet cross grain size, which is around $100 \mathrm{~nm}$ (Fig. 8d). Indeed, the detailed nanotubes micrographs, Fig. 8b and c, revealed horizontal stripes with respect to the nanotubes length (see the white arrow in Fig. $8 \mathrm{~b}$ and $\mathrm{c}$ ), which match the Ta cross grain size. 


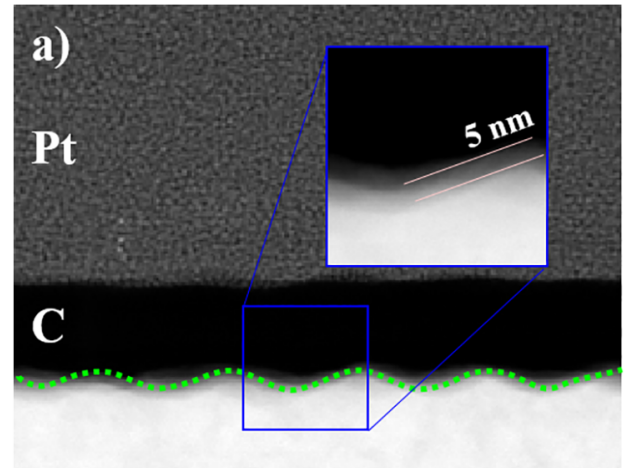

HAADF

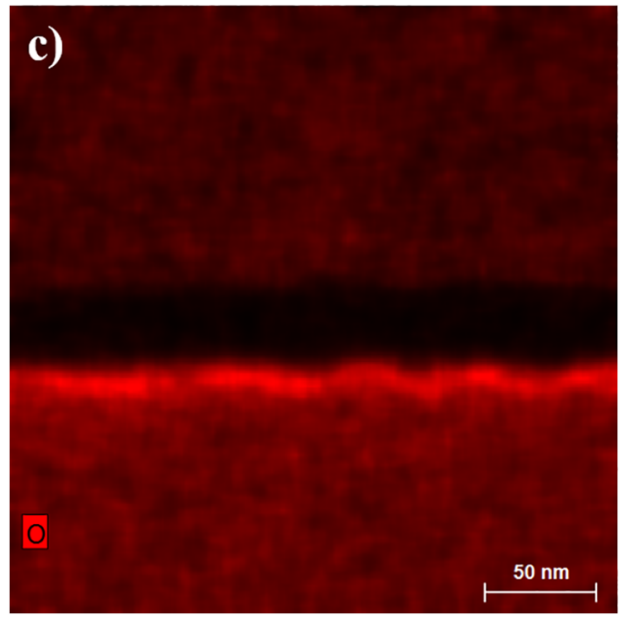

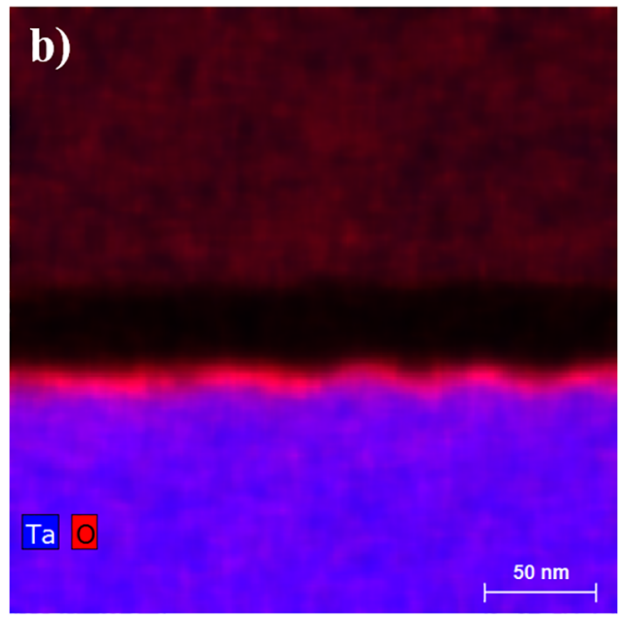

d)

$\mathrm{Ta}$ $50 \mathrm{~nm}$

Fig. 4. (a) Cross-section HAADF STEM image and (b-d) EDS spectrum images showing the elemental distribution for the anodic layer of a Ta sample anodized with 8:0.5 (vol:vol) of $\mathrm{H}_{2} \mathrm{SO}_{4}: \mathrm{HF}$, at room temperature under $15 \mathrm{~V}$ during $120 \mathrm{~s}$.

Moreover, the presence of $\mathrm{S}$ and $\mathrm{F}$ in the nanotube walls and their absence in the dimples surface demonstrates that the water-solubility exhibited by the metal-fluoride complexes incorporated into the anodic layer may enhance the nanotubes release during the rinse of the samples with distilled water. The nanotubes removal leaves behind a surface with ordered dimple-shaped morphology. The complete description of the process is schematized in Fig. 9.

Nonetheless, the size and distribution of these nanostructures can be controlled using the anodization potential, since the electric field distribution along the surface determines the nanostructures diameter

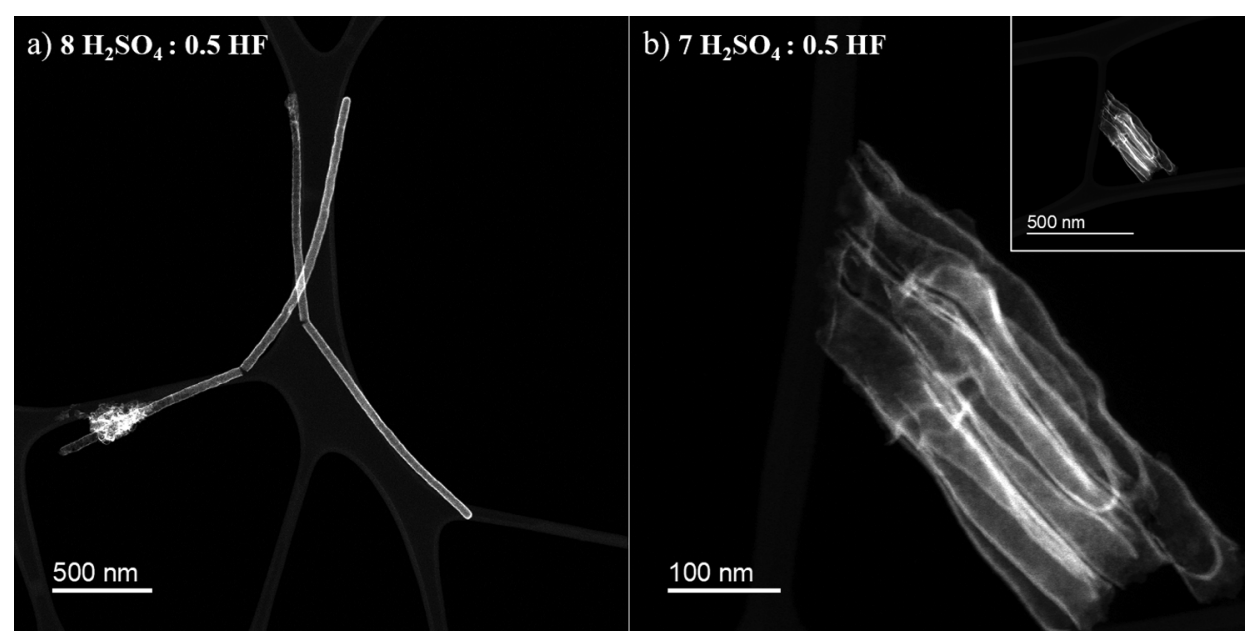

Fig. 5. HAADF STEM images of the $\mathrm{TaO}_{\mathrm{x}}$ anodic nanotubes formed during anodization at room temperature under $15 \mathrm{~V}$ during $120 \mathrm{~s}$ with: (a) 8:0.5 (vol:vol) of $\mathrm{H}_{2} \mathrm{SO}_{4}$ :HF; and (b) 7:0.5 (vol:vol) of $\mathrm{H}_{2} \mathrm{SO}_{4}: \mathrm{HF}$. 


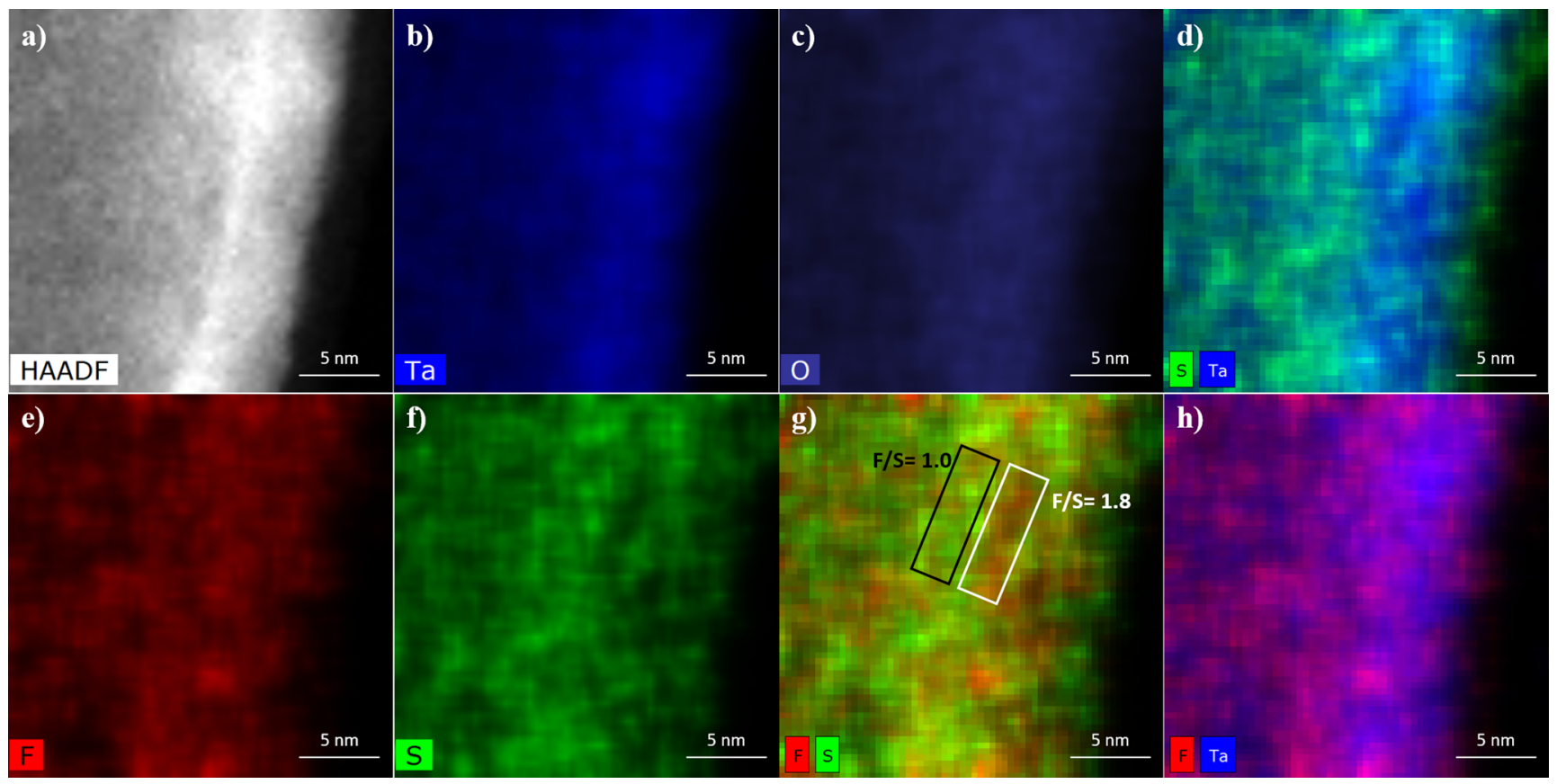

Fig. 6. (a) HAADF STEM and (b, c, d, e, f, g and h) EDS spectrum images showing the elemental distribution for the anodic nanotubes wall present in the solid residues of the electrolyte after anodization with 8:0.5 (vol:vol) of $\mathrm{H}_{2} \mathrm{SO}_{4}: \mathrm{HF}$, at room temperature under $15 \mathrm{~V}$ during $120 \mathrm{~s}$. The area underlined by the black and white boxes in $(\mathrm{g})$ was used to estimate the $\mathrm{F} / \mathrm{S}$ ratio using the EDS spectrum of each specific region.

[15]. In this regard, in order to study the influence of the anodization potential on the diameter of the dimples, Ta anodized surfaces were prepared with 8:0.25 (vol:vol) mixture of $\mathrm{H}_{2} \mathrm{SO}_{4}$ with $\mathrm{HF}$, at room temperature during $120 \mathrm{~s}$ and for voltages in the range of $15-50 \mathrm{~V}$. The lower electrolyte concentration, above the dissolution limit was selected in an attempt to have higher control on the process, as well as, a
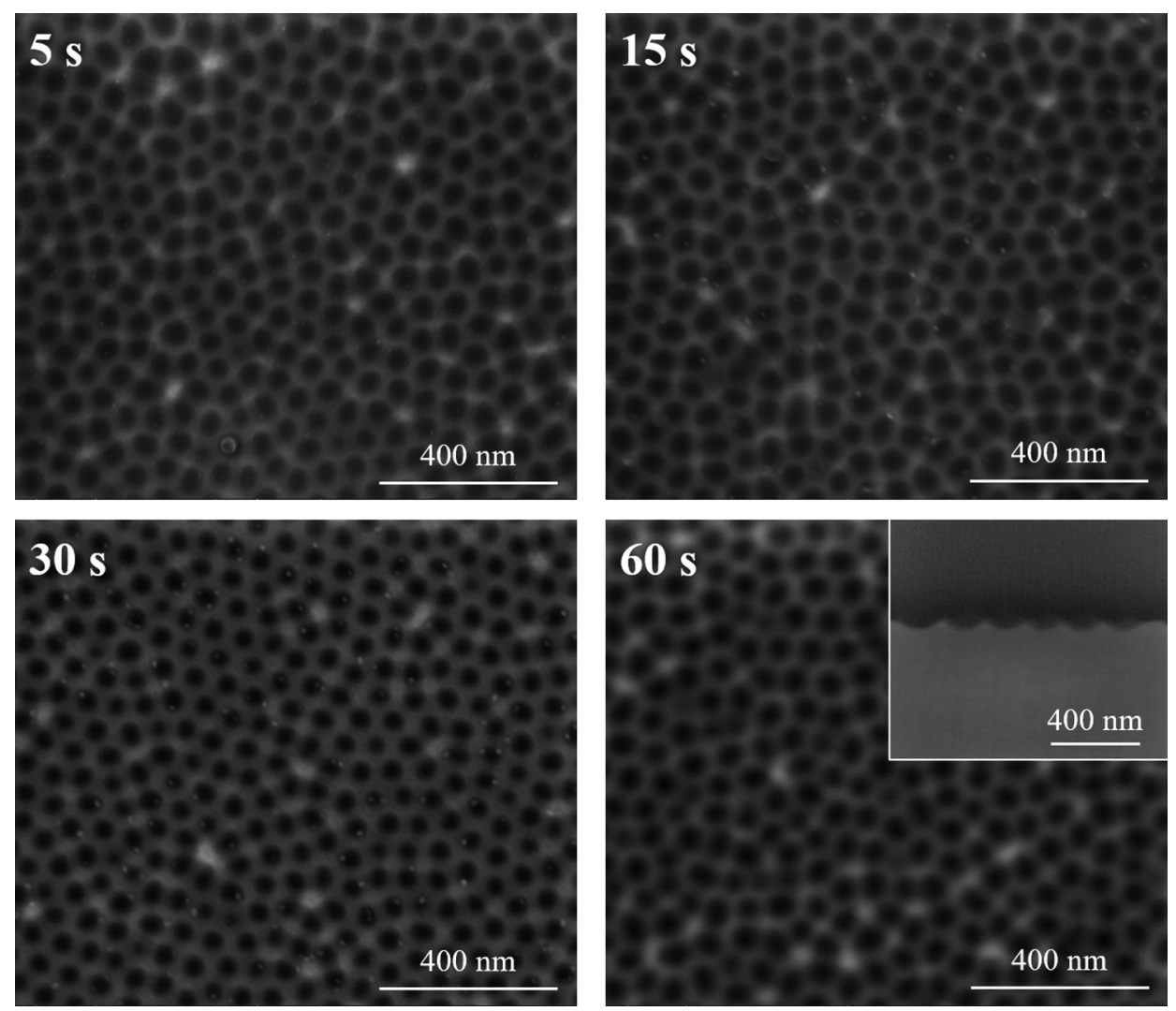

Fig. 7. Secondary electron SEM images of Ta anodized surfaces with 8:0.25 (vol:vol) of $\mathrm{H}_{2} \mathrm{SO}_{4}: \mathrm{HF}$, at room temperature under $25 \mathrm{~V}$ as a function of the anodization time $(5-60 \mathrm{~s})$. 

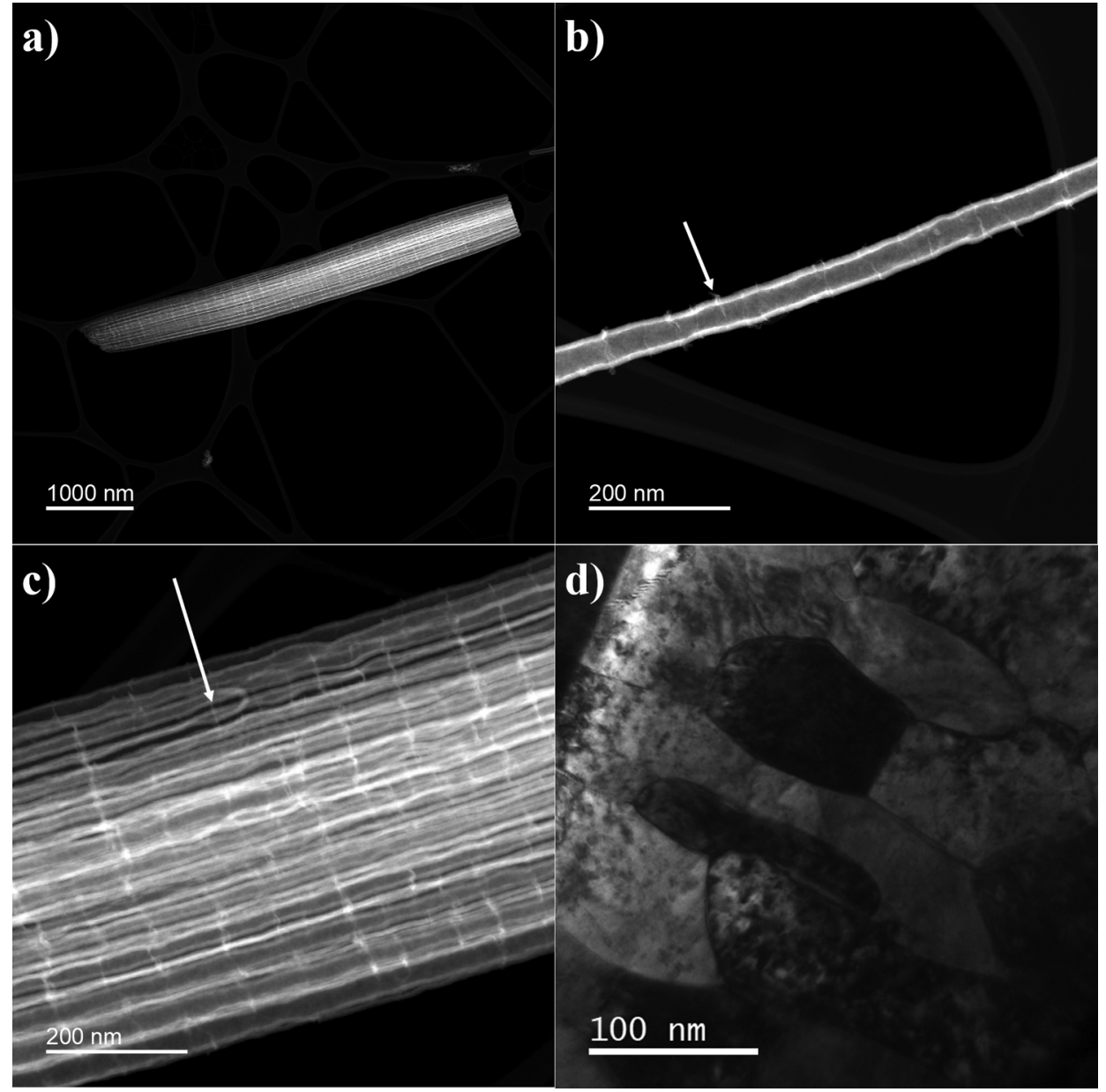

Fig. 8. (a) (b) and (c) Representative HAADF STEM image of the $\mathrm{TaO}_{\mathrm{x}}$ anodic nanotubes formed during anodization; (d) Cross-section bright-field TEM image of Ta sheet.

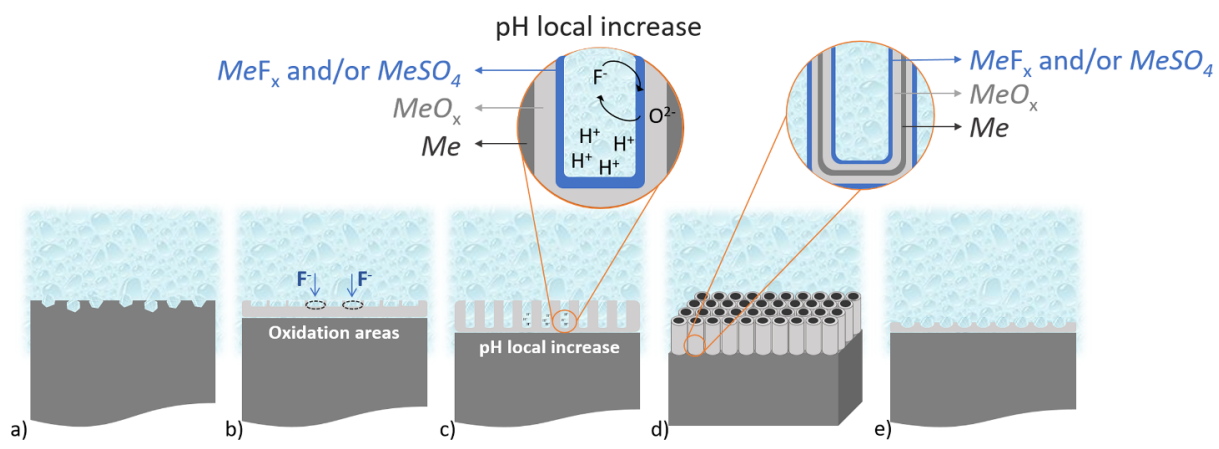

Fig. 9. Schematic representation of the nanopore-nanotube assembly' mechanism: (a) Surface with pits before anodization; (b) Dissolution of the $\mathrm{Ta}_{2} \mathrm{O}_{5}$ anodic layer and formation of dimples; (c) Growth of nanopores (length grown by electric field); (d) Formation of nanotubes; and (e) Dimple-shaped morphology.

higher control of the morphology. The effect of the applied voltage on the dimples diameter is shown in Fig. 10. The number of dimples in the micrographs is clearly reduced when the anodization potential increases. Moreover, as the potential increases a broader size distribution is reached. Hence, increasing the electrical field, leads to more differential dissolution, as a consequence of the faster electrical field spread and dissolution rate in random modulations existent on the sample surface.

Initially the distribution of the electric field is not uniform due to the asperities and therefore, there is preferential dissolution on the asperities. Then, due to the polishing effect, the field becomes more uniform and thus, ordered structures are obtained. Also, knowing that the nanostructures grow from an asperity in the Ta surface, we show that the growth of the nanostructures takes place in all directions due to the hemispherical spread of the electric field and hence, the oxide structures acquire a hemispherical shape [23]. This demonstrates that the anodization potential plays a significant role in controlling the nanostructure diameter. 
a)

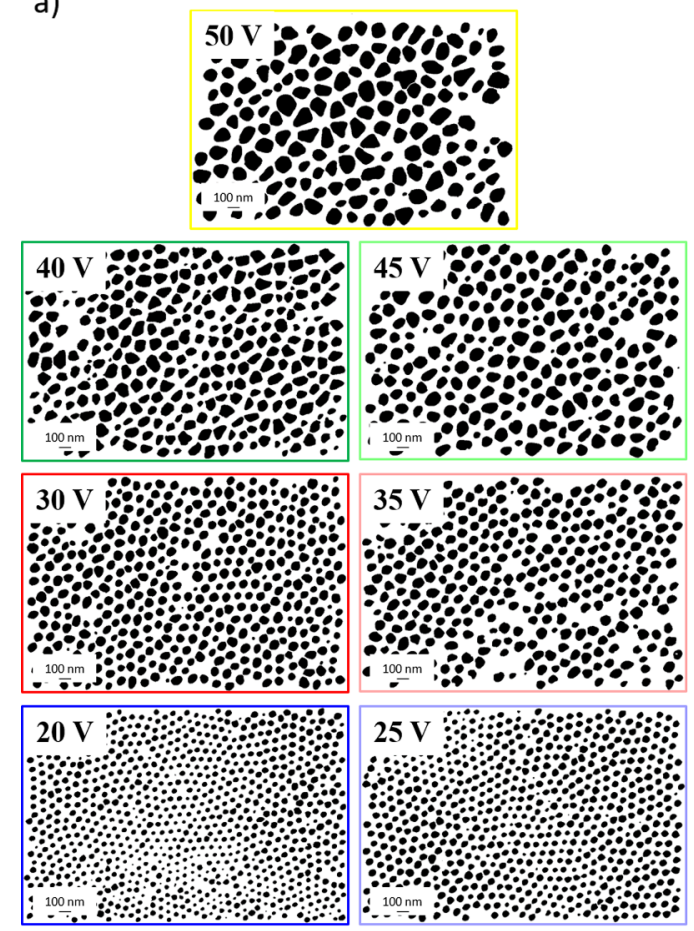

b)

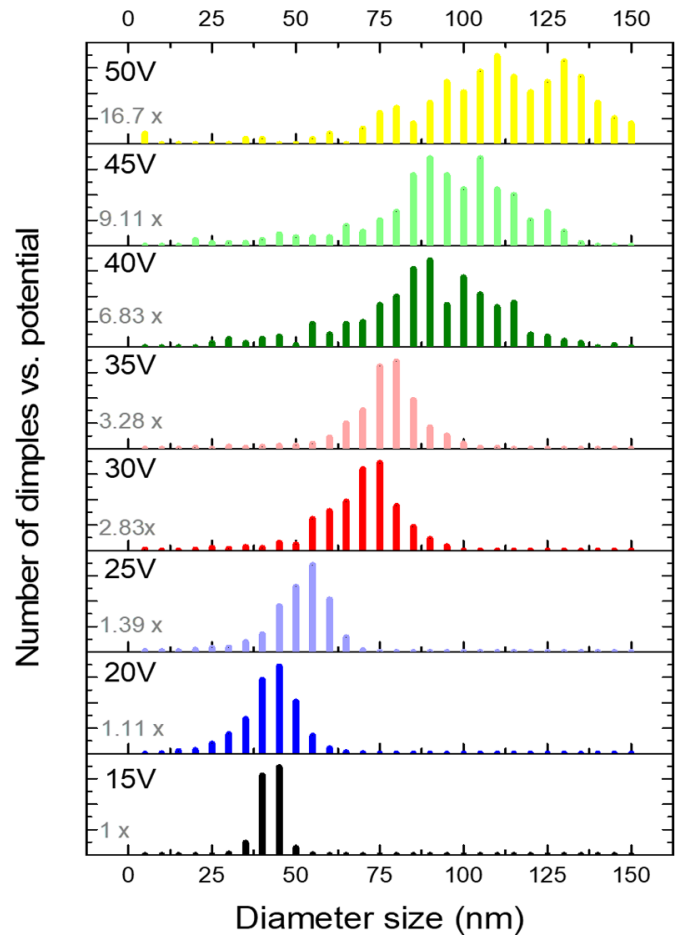

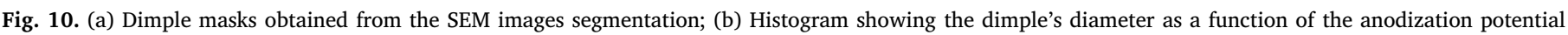
(15-50 V) applied to the Ta anodized samples.

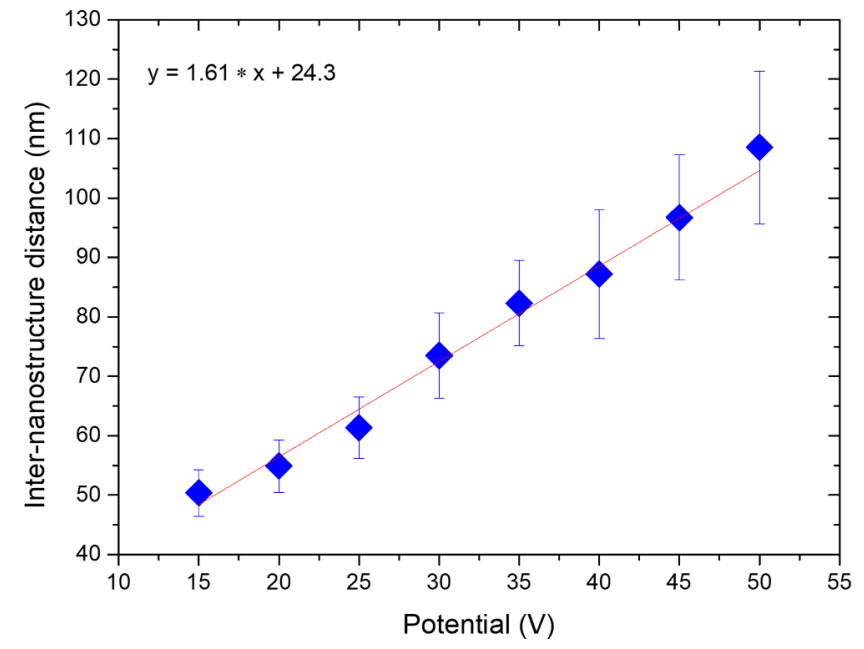

Fig. 11. Dimples' first neighbor distance as function of applied voltage of Ta anodized surfaces with 8:0.25 (vol:vol) of $\mathrm{H}_{2} \mathrm{SO}_{4}: \mathrm{HF}$, at room temperature during $120 \mathrm{~s}$.

The nanostructures diameter is linearly dependent of the anodization voltage applied during the process, as previously reported [18]. Fig. 11 shows the first neighbor distance as a function of the applied potential (from $15 \mathrm{~V}$ up to $50 \mathrm{~V}$ ) of Ta anodized surfaces with 8:0.25 (vol:vol) mixture of $\mathrm{H}_{2} \mathrm{SO}_{4}$ with $\mathrm{HF}$, at room temperature during $120 \mathrm{~s}$.

Indeed, the first neighbor distances follow a linear trend, which can be empirically correlated with the applied potential. Hany El-Sayed et al. [15] reported a similar trend for an 9:1 (vol:vol) mixture of $\mathrm{H}_{2} \mathrm{SO}_{4}$

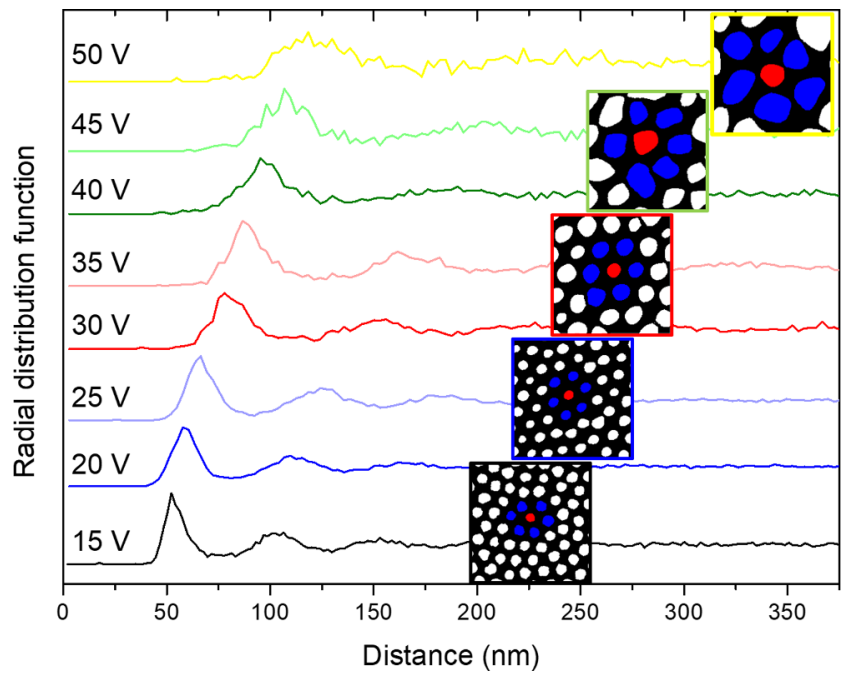

Fig. 12. Dimples' radial distribution function as function of applied voltage of Ta anodized surfaces with 8:0.25 (vol:vol) of $\mathrm{H}_{2} \mathrm{SO}_{4}: \mathrm{HF}$, at room temperature during $120 \mathrm{~s}$. The inset images correspond to the filtered false-colored SEM images presenting the coordination number for each condition.

with $\mathrm{HF}$, at room temperature during 10 min under $10-20 \mathrm{~V}$, and found a "growth factor" with a voltage of about $1.5 \mathrm{~nm} / \mathrm{V}$, which is very close of the experimental value estimated in this study $1.6 \mathrm{~nm} / \mathrm{V}$.

The long-range order is also affected by the electric field. In this regard, in order to study the influence of the electric field on long-range order, Fig. 12 shows the RDF and the coordination number as a function 
of the applied potential (from $15 \mathrm{~V}$ up to $50 \mathrm{~V}$ ) of Ta anodized surfaces with 8:0.25 (vol:vol) mixture of $\mathrm{H}_{2} \mathrm{SO}_{4}$ with $\mathrm{HF}$, at room temperature during $120 \mathrm{~s}$.

With an increase of the electric field, the long-range order is decreased. As mentioned above, increasing the applied potential promotes a differential dissolution, creating a larger dispersion and distribution of the pores, which leads to a disordered structure. This phenomenon is also in good agreement with the loss in circularity by the nanostructures as shown in Fig. 10a.
The authors thank the financial support in the framework of HEALTHYDENT - POCI-01-0145-FEDER-030708 and PTDC/CTM-NAN/ $4242 / 2014$ projects.

This work was supported by FCT, through IDMEC, under LAETA, project UIDB/50022/2020.

The authors would like to acknowledge that this project has received funding from the EU Framework Programme for Research and Innovation H2020, scheme COFUND - Co-funding of Regional, National and International Programmes, under Grant Agreement 713640.

\section{DOQH Q $\mathrm{QP}=0$}

\section{Conclusions}

In this work, we demonstrate the influence of the $\mathrm{H}_{2} \mathrm{SO}_{4}$ concentration to achieve ordered dimple nanostructures. The decrease of the oxide dissolution rate for higher $\mathrm{H}_{2} \mathrm{SO}_{4}$ concentrations and $\mathrm{SO}_{4}{ }^{2-}$ ion incorporation into the anodic nanoarrays layer enhances the oxide dissolution rate by $\mathrm{F}^{-}$ions and causing its detachment from the surface. In addition, decreasing the HF concentration (down to 0.125) leads to a dissolution limit of the anodic $\mathrm{TaOx}$, which compromises the dissolution rate and affects the nanoarrays order.

For all the different parameters used (electrolyte concentration, applied voltage, anodization time) a dimpled-shaped morphology is achieved. The absence of $\mathrm{S}$ and $\mathrm{F}$ in the dimple composition and the presence of $\mathrm{S}$ and $\mathrm{F}$ into the nanotube walls suggests the formation on the surface of unstable nanotubes that can be easily removed by water cleaning.

The size and distribution of these nanostructures can be controlled using the anodization potential, due to modifications of the electrical field during anodization. The increase of the anodization potential/ electrical field leads to an increase in the dimple average diameter. Additionally, increasing the electrical field results in a more differential dissolution, as a consequence of the faster electrical field spread promoting a larger dispersion and distribution of diameters, which leads to a disordered structure.

\section{CRediT author statement}

All authors have contributed for the conceptualization, methodology, formal analysis and funding acquisition of the present review paper. C. F. Almeida Alves wrote the original document that was reviewed and edited by S. Calderon V., P. J. Ferreira, L. Marques and S. Carvalho. C. F. Almeida Alves performed the review visualization. S. Carvalho supervised the research.

\section{Declaration of Competing Interest}

The authors declare that they have no known competing financial interests or personal relationships that could have appeared to influence the work reported in this paper.

\section{Acknowledgements}

This research is sponsored by FEDER funds through the program COMPETE - Programa Operacional Factores de Competitividade and by the Portuguese Foundation for Science and Technology (FCT) in the framework of the Strategic Funding UIDB/04650/2020, and UID/EMS/ 00285/2020 and with a PhD fellowship SFRH/BD/98199/2013.

\section{References}

[1] S. Singh, W.R.T. Barden, P. Kruse, Nanopatterning of transition metal surfaces via electrochemical dimple array formation, ACS Nano 2 (2008) 2453-2464.

[2] H. El-Sayed, S. Singh, M.T. Greiner, P. Kruse, Formation of highly ordered arrays of dimples on tantalum at the nanoscale, Nano Lett. 6 (2006) 2995-2999.

[3] I. Sieber, H. Hildebrand, A. Friedrich, P. Schmuki, Initiation of tantalum oxide pores grown on tantalum by potentiodynamic anodic oxidation, J. Electroceramics. 16 (2006) 35-39.

[4] H.A. El-Sayed, V.I. Birss, Controlled interconversion of nanoarray of Ta dimples and high aspect ratio Ta oxide nanotubes, Nano Lett. 9 (2009) 1350-1355.

[5] M. Sowa, A. Kazek-k, R.P. Socha, G. Dercz, J. Michalska, W. Simka, Modification of tantalum surface via plasma electrolytic oxidation in silicate solutions, Electrochim. Acta. 114 (2013) 627-636, https://doi.org/10.1016/j.electacta.2013.10.047.

[6] H.A. Acciari, D.P.S. Palma, E.N. Codaro, Q. Zhou, J. Wang, Y. Ling, J. Zhang, Z. Zhang, Surface modifications by both anodic oxidation and ion beam implantation on electropolished titanium substrates, Appl. Surf. Sci. 487 (2019) 1111-1120, https://doi.org/10.1016/j.apsusc.2019.05.216.

[7] N.K. Allam, X.J. Feng, C.A. Grimes, Self-assembled fabrication of vertically oriented $\mathrm{Ta}_{2} \mathrm{O}_{5}$ nanotube arrays, and membranes thereof, by one-step tantalum anodization, Chem. Mater. 20 (2008) 6477-6481.

[8] S. Boughaba, M.U. Islam, G.I. Sproule, M.J. Graham, Characterization of tantalum oxide films grown by pulsed laser deposition, Surf. Coatings Technol. 120 (1999) 757-764.

[9] H.-E. Cheng, C.-T. Mao, The effect of substrate temperature on the physical properties of tantalum oxide thin films grown by reactive radio-frequency sputtering, Mater. Res. Bull. 38 (2003) 1841-1849.

[10] L. Yang, M. Viste, J. Hossick-Schott, B.W. Sheldon, Internal stress evolution during field-induced crystallization of anodic tantalum oxide, Electrochim. Acta. 81 (2012) 90-97.

[11] A. Al-masha'al, A. Bunting, R. Cheung, Evaluation of residual stress in sputtered tantalum thin-film, Appl. Surf. Sci. 371 (2016) 571-575. doi: https://doi.org/10 1016/j.apsusc.2016.02.236

[12] Y. Liu, C. Bao, D. Wismeijer, G. Wu, The physicochemical/biological properties of porous tantalum and the potential surface modification techniques to improve its clinical application in dental implantology, Mater. Sci. Eng. C. 49 (2015) 323-329.

[13] W. Wei, J.M. Macak, N.K. Shrestha, P. Schmuki, Thick self-ordered nanoporous $\mathrm{Ta}_{2} \mathrm{O}_{5}$ films with long-range lateral order, J. Electrochem. Soc. 156 (2009) K104-K109.

[14] H.A. El-Sayed, V.I. Birss, Controlled growth and monitoring of tantalum oxide nanostructures, Nanoscale. 2 (2010) 793-798.

[15] H.A. El-Sayed, H.M. Molero, V.I. Birss, The impact of fabrication conditions on the quality of Au nanoparticle arrays on dimpled Ta templates, Nanotechnology. 23 (2012) 435602.

[16] J.E. Barton, C.L. Stender, P. Li, T.W. Odom, Structural control of anodized tantalum oxide nanotubes, J. Mater. Chem. 19 (2009) 4896-4898.

[17] W. Chen, Q. Tu, H. Wu, C. Zhao, X. Yao, W. Fan, S. Zhang, J. Ni, X. Zhang, Study on morphology evolution of anodic tantalum oxide films in different using stages of $\mathrm{H}_{2} \mathrm{SO}_{4} / \mathrm{HF}$ electrolyte, Electrochim. Acta. 236 (2017) 140-153.

[18] L.-N. Wang, M. Jin, Y. Zheng, Y. Guan, X. Lu, J.-L. Luo, Nanotubular surface modification of metallic implants via electrochemical anodization technique, Int. J. Nanomedicine. 9 (2014) 4421.

[19] H. El-Sayed, S. Singh, P. Kruse, Formation of dimpled tantalum surfaces from electropolishing, J. Electrochem. Soc. 154 (2007) C728-C732.

[20] A. Aspart, C.Z. Antoine, Study of the chemical behavior of hydrofluoric, nitric and sulfuric acids mixtures applied to niobium polishing, Appl. Surf. Sci. 227 (2004) 17-29, https://doi.org/10.1016/j.apsusc.2003.10.001.

[21] M. Lippold, S. Patzig-Klein, E. Kroke, HF-HNO3-H2SO4/H2O mixtures for etching multicrystalline silicon surfaces: formation of $\mathrm{NO}_{2}+$, reaction rates and surface morphologies, Zeitschrift Für Naturforsch. B. 66 (2011) 155-163. 
[22] D.A. Vermilyea, The formation of anodic oxide films on tantalum in non-aqueous solutions, Acta Metall. 2 (1954) 482-486.

[23] A. Ghicov, P. Schmuki, Self-ordering electrochemistry: a review on growth and functionality of $\mathrm{TiO}_{2}$ nanotubes and other self-aligned $\mathrm{MO}$ x structures, Chem. Commun. 2791-2808 (2009).
[24] K. Lee, A. Mazare, P. Schmuki, One-dimensional titanium dioxide nanomaterials: nanotubes, Chem. Rev. 114 (2014) 9385-9454.

[25] H.A. El-Sayed, C.A. Horwood, A.D. Abhayawardhana, V.I. Birss, New insights into the initial stages of Ta oxide nanotube formation on polycrystalline Ta electrodes, Nanoscale. 5 (2013) 1494-1498. 